\title{
FUNCTIONAL WARP-KNITTED FABRICS WITH INTEGRATED SUPERELASTIC NITI FILAMENTS
}

\author{
Katerina Janouchova', Ludek Heller², Monika Vysanska
}
1Department of Textile Technologies, Faculty of Textile Engineering, Technical University of Liberec, Czech Republic, katerina.janouchova1@tul.cz, monika.vysanska@tul.cz
2Department of Functional Materials, Institute of Physics of the AS CR Prague, Czech Republic, heller@fzu.cz

\begin{abstract}
:
We report on a particular direction of currently conducted extended research on novel textiles with integrated thin metallic filaments made of an intermetallic shape memory NiTi alloy exhibiting functional behaviour such as superelastic deformation up to $10 \%$ and a thermally induced shape memory effect. Within this research direction we focus on development of single and multi-layered warp-knitted fabrics that are directionally reinforced with superelastic NiTi filaments. First, we describe the expected properties of such novel structures and their potential applications. Second, we present the functional thermomechanical behaviour of applied superelastic NiTi filaments. Third, we address questions related to the design and fabrication of warp-knitted fabrics with integrated NiTi filaments. Then, we describe experimental methods applied on novel functional textiles in order to evaluate their functional properties. Finally, we present and discuss results of experiments carried out on these novel functional textiles.
\end{abstract}

\section{Key words:}

Warp knitting, phase change materials, shape memory alloys, superelasticity, martensitic transformation, Nitinol.

\section{Introduction}

The use of shape memory materials represents one of several approaches which are being adopted in the development of smart textiles that exhibit additional functional properties and/ or outstanding structural behaviour [1]. Within this approach, shape memory polymers (SMP) are most commonly used, as their structural and other comfort properties (hand, air permeability, surface roughness, drape, etc.) are similar to those of conventional textile materials [1]. In contrast, the use of shape memory alloys (SMA) in smart textiles has been reported to a much smaller extent [2, 3], though they outperform SMP in many aspects such as strength, stability of thermal and cyclic properties, thermally induced recovery stresses, etc. [4]. In addition, incorporation of SMA into textiles has been mostly limited to their ex-post integration in the form of coils or ribbons into conventional textiles [1]. However, development of hybrid SMA textiles produced by direct textile processing of SMA filaments has not been addressed extensively.

In this paper, we deal with the design, fabrication and evaluation of mechanical properties of single and architectured multilayered warp-knitted fabrics made of conventional textile materials and incorporating straight superelastic SMA filaments in the weft direction. Such fabric has been conceived in order to project the functional properties of SMA filaments onto a plain structure and combine these properties with the structural behaviour of conventional weft-knitted fabrics. Hence, in such a design, the weft-knitted structure provides not only structural support for SMA filaments but also mechanical properties that are responsible for macroscopic behaviour when the loading direction is not parallel to the orientation of the SMA filaments. Moreover, we extend this design to multilayered structures comprising several single weft-knitted fabrics stacked and fixed to each other at different mutually rotated NiTi filaments orientations (i.e. an architectured structure) so as to provide a desired degree of anisotropy of functional properties inherited from SMA filaments.
These single and architectured multi-layered warp-knitted fabrics might be used for applications which would benefit from the following functional properties:

- Functional properties of superelastic SMA wires

- Superelasticity (see below),

- High strength ( 2 GPa),

- High passive damping capacity,

- Customized directional distribution of functional properties.

\section{Functional behaviour of thin superelastic niti filaments}

Shape memory alloys belong to the class of smart materials whose functional properties are derived from reversible solidstate thermoelastic martensitic transformation [5]. They exhibit functional properties such as the temperature driven one- and two-way memory effect, superelastic deformation (up to $10 \%$ strain), high damping capacity and high work output upon a thermal excitation. Among these alloys, NiTi is currently the most popular SMA and is being commercially used mainly in the biomedical field, as NiTi has been proven biocompatible [6]. Besides, NiTi alloy shows by far the largest energy density, as it exhibits simultaneously large recoverable strains and stresses during thermal actuation.

In this work, we selected thin superelastic NiTi filaments 75 microns in diameter as the material providing the functional properties to be projected onto the weft-knitted textiles. We purchased these filaments from Fort Wayne Metals Ltd. [7], who provide them under the product name NiTi \#1. The filaments were delivered in so-called straight-annealed state, i.e., they exhibited functional properties in as-delivered state and no further heat treatment had to be applied. The selected $\mathrm{NiTi}$ alloy is called superelastic as the stable microstructure phase at room temperature is cubic austenite, which can be transformed into monoclinic martensite, which is stable at lower temperatures, by applying external loads. This so-called 
stress-induced martensitic transformation gives rise to nonlinear hysteretic tensile behaviour, as shown in Fig. 1, which depicts all the peculiar features of superelastic NiTi filaments, such as large recoverable strain (8\%), occurrence of plateaus, different Young module of austenite ( $50 \mathrm{GPa})$ and martensite ( 20 GPa), stress hysteresis and strong thermomechanical coupling that shifts the transformation plateaus by $5.5 \mathrm{MPa}$ per degree of temperature change. Besides these functional properties, the filaments show excellent mechanical properties such as an ultimate tensile strength of $1.8 \mathrm{GPa}$ and yield stress for plasticity of $1.6 \mathrm{GPa}$ [4].

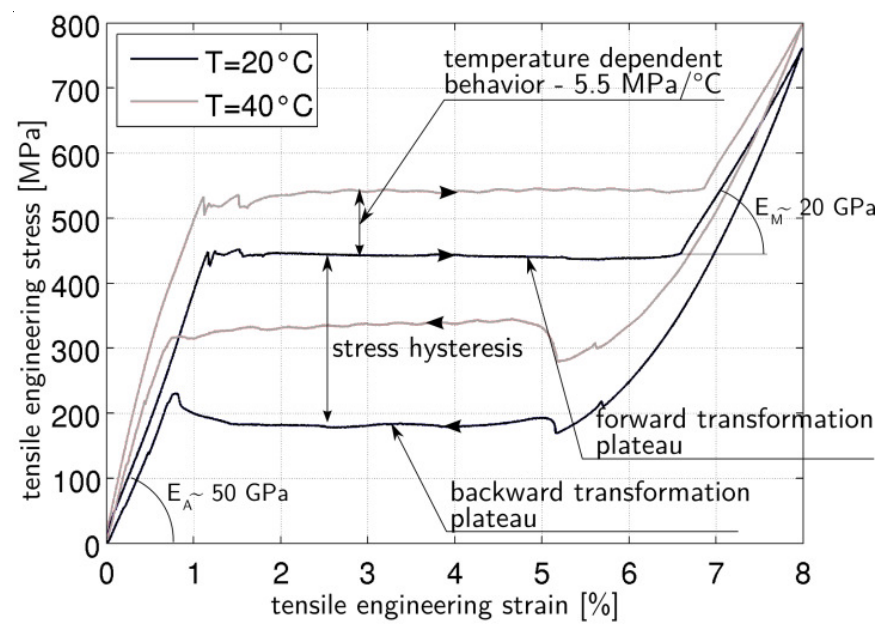

Figure 1. Tensile behaviour of the used superelastic NiTi filaments of diameter $0.075 \mathrm{~mm}$ at two temperatures.

\section{Design and manufacture of functional warp- knitted fabrics}

Functional warp-knitted fabrics are based on warp-knitted closed fabrics with wale density of $500 \mathrm{~m}^{-1}$ and course density of $800 \mathrm{~m}^{-1}$ made of a polyester multifilament yarn of 38 tex. Firstly, a reference sample of such a fabric was manufactured. In this paper, the reference sample is denoted as PES. Secondly, the functional warp-knitted fabric was manufactured by inserting thermoplastics-coated NiTi filaments in the course direction every 5th course during warp knitting. The thermoplastic coating of NiTi filaments was applied in order to fix NiTi filaments in the fabric. The fixation was realized after knitting through application of heat and pressure on the fabric, which led to gluing the NiTi filaments to polyester yarns through melted thermoplastic coating. Fig. 2 shows the structure of the manufactured functional warp-knitted fabric. In this paper, the functional warp-knitted fabric sample is referred to as $\mathrm{NiTi}+\mathrm{PES}$.

\section{Experimental characterization of mechanical properties of functional warp-knitted fabrics}

We evaluated the mechanical functional behaviour of the studied warp-knitted fabrics using two experimental methods. First, we applied simple uniaxial tensile tests at room temperature to investigate the mechanical behaviour under uniaxial loads. During a tensile test, we recorded the tensile force, displacement of grips, and the displacement field on the surface of the fabric using the technique described in the next paragraph. Second, we employed dedicated in-house made bulge test equipment with an aperture of $100 \mathrm{~mm}$ diameter (see its scheme in Fig. 3 and photograph in Fig. 4) to investigate the mechanical behaviour under complex loads. When applying the bulge test, the specimen is placed over a circular die with a spherical aperture and clamped around the

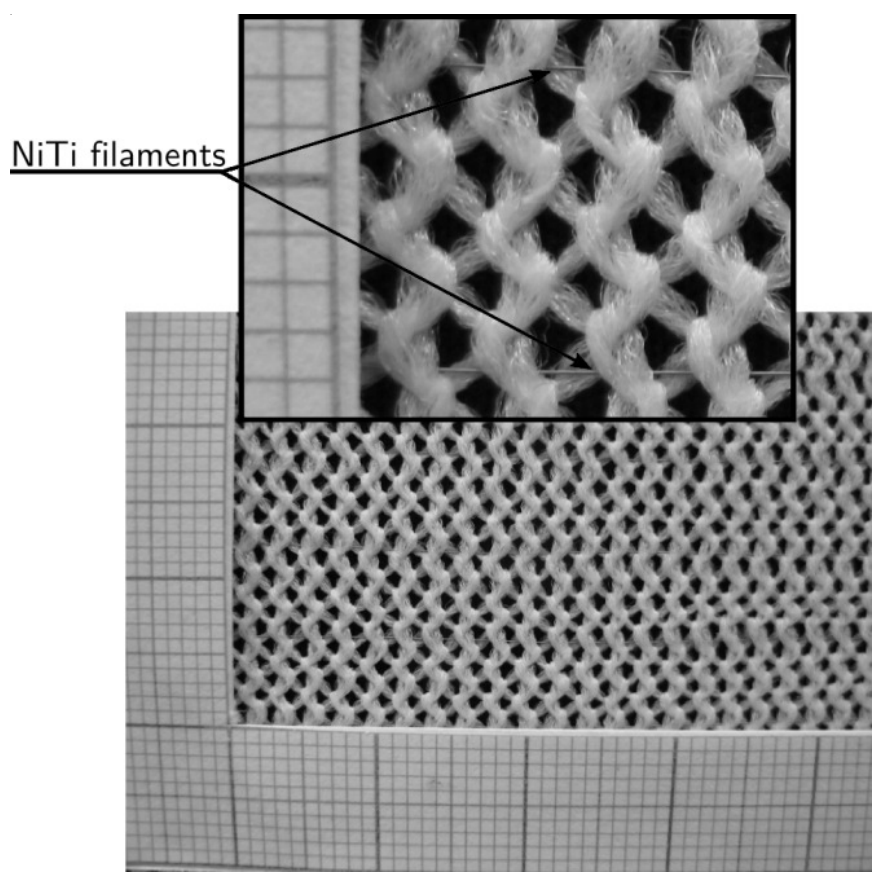

Figure 2. Photographs of the functional warp-knitted fabric sample referred to as $\mathrm{NiTi+PES}$.

perimeter. Then, an increasing pressure is applied to the inner side of the diaphragm, causing the specimen to bulge through the aperture. During a bulge test, we recorded evolution of inner pressure, stroke of the pole of the deformed specimen and the displacement field on the surface of the fabric using the technique described below. As the studied textiles are porous, they were underlaid with a thin elastomeric membrane. Therefore, the bulge test in such a configuration provides data that is somehow distorted due to the presence of the membrane. However, the stiffness of the membrane is extremely low, as can be seen in Fig. 5, comparing data measured on the membrane only and reference warp-knitted fabric with the underlying membrane. It can be seen that the stiffness of the membrane is far lower than that of the reference warp-knitted fabric, which itself is much softer than functional
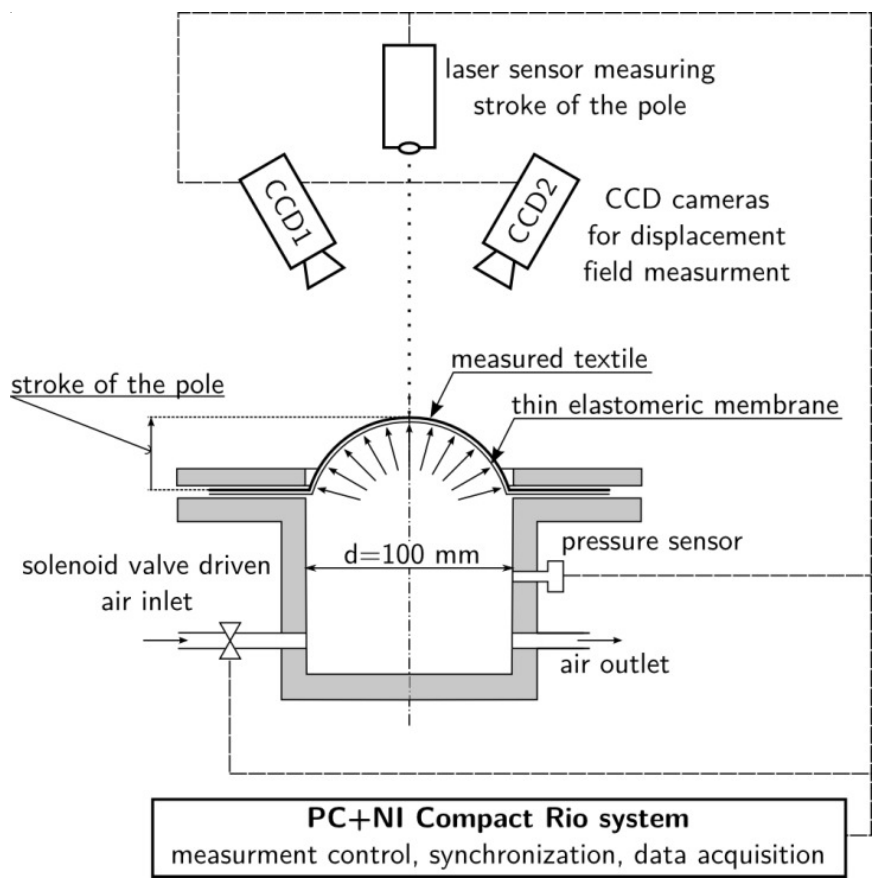

Figure 3. Schematic representation of the bulge tester showing its main components. 

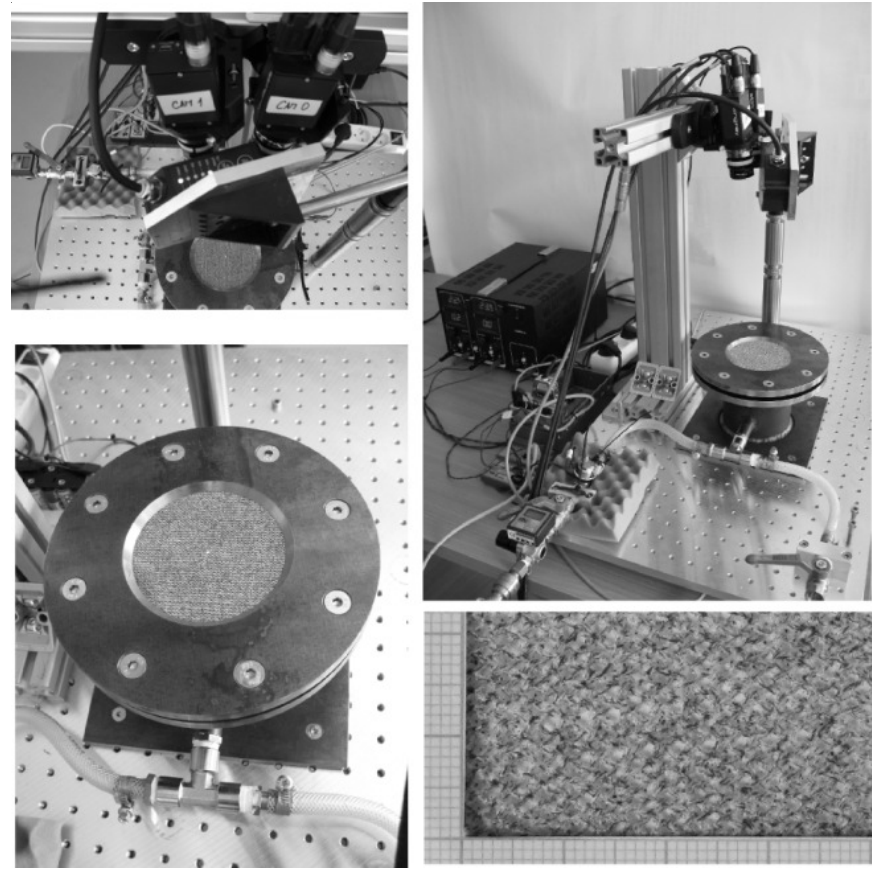

Figure 4. Photographs of the bulge tester and details of a specimen of a tested textile with painted speckle pattern for digital image correlation.

warp-knitted fabric, as will be seen in the following text. Therefore, we neglect the distorting effect of the membrane on experimental data provided by the bulge tester.

Both the methods were applied in a quasi-static loading regime and in conjunction with the use of the digital image correlation (DIC) technique, allowing for measurement of the displacement field at the surface of the fabrics. The technique consists of painting a speckle pattern (see Fig. 4) on the fabric and recording the photographs of the pattern during mechanical loading of the fabric. The displacement field is then computed through the correlation between patterns recorded at different loading steps.

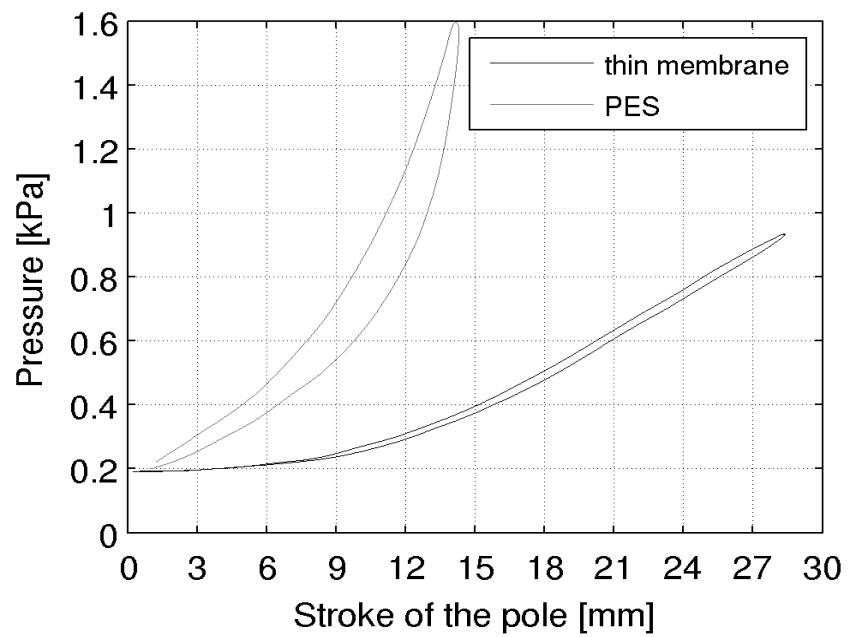

Figure 5. Bulge test results obtained on underlying thin membrane and polyester weft-knitted fabric (PES) underlaid with the thin membrane.

\section{Presentation and discussion of experimental results}

Mechanical behaviour under uniaxial tensile loads

A reference PES sample free of NiTi filaments and a functional warp knitted fabric sample denoted as $\mathrm{NiTi}+\mathrm{PES}$ containing

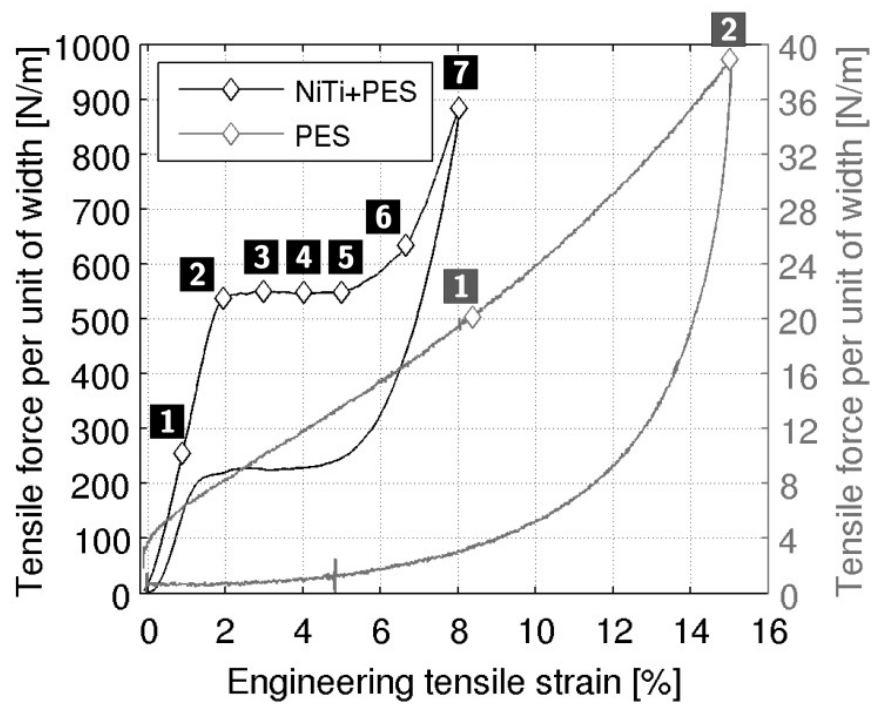

Figure 6. Experimental tensile curves related to uniaxial strokecontrolled loading/unloading ramps in course direction, i.e., along the direction of NiTi filaments in the case of the NiTi+PES sample. The $\mathrm{NiTi}+\mathrm{PES}$ sample contained $26 \mathrm{NiTi}$ filaments. PES denotes the reference sample free of NiTi filaments. Numbered markers represent instants at which spatial displacement fields were measured.

$26 \mathrm{NiTi}$ filaments were subjected to uniaxial stroke-controlled loading/unloading ramps in the course direction, i.e., along the direction of the NiTi filaments. Fig. 6, displaying related experimental tensile curves, shows clearly that the tensile responses of both the samples differ qualitatively as well as quantitatively.

First, the tensile response of the $\mathrm{NiTi}+\mathrm{PES}$ sample is completely driven by the tensile properties of NiTi samples (compare the tensile curve for $\mathrm{NiTi+PES}$ in Fig. 6 with that in Fig. 1), whereas the tensile response of the PES sample corresponds to the one typically observed on conventional knitted fabrics. Moreover, not only is the macroscopic tensile response of the $\mathrm{NiTi}+\mathrm{PES}$ sample inherited from the tensile behaviour of NiTi filaments but their presence also gives rise to the phenomenon of localized transformation deformation, which is the typical deformation mechanism in SMAs. The appearance of localized deformation can be revealed as an inhomogeneity in the spatial displacement derivative. Such an

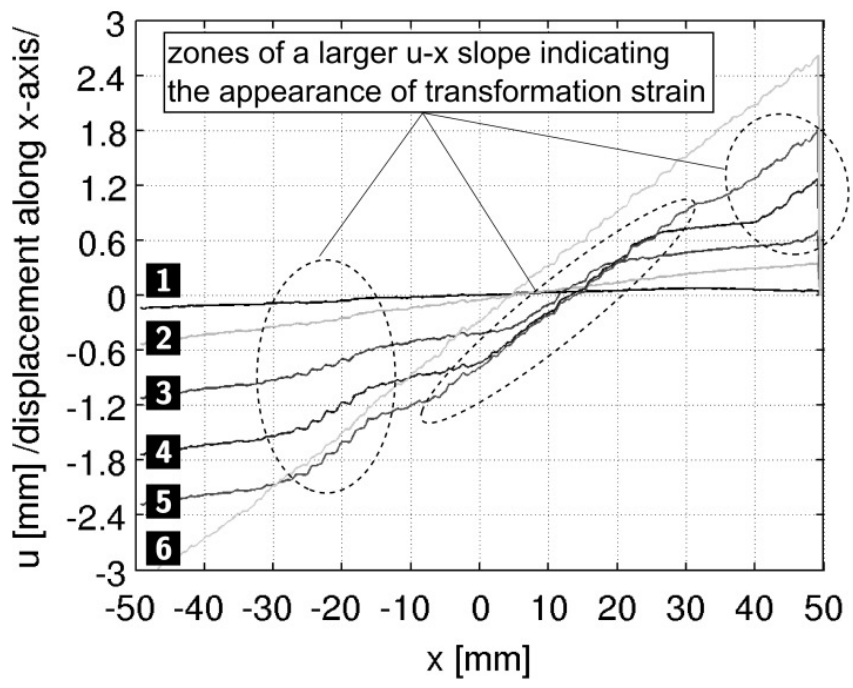

Figure 7. Displacement in the $x$ direction along the sample axis (see Fig. 8) of the NiTi+PES sample measured at several loading instants according to Figure 6. 


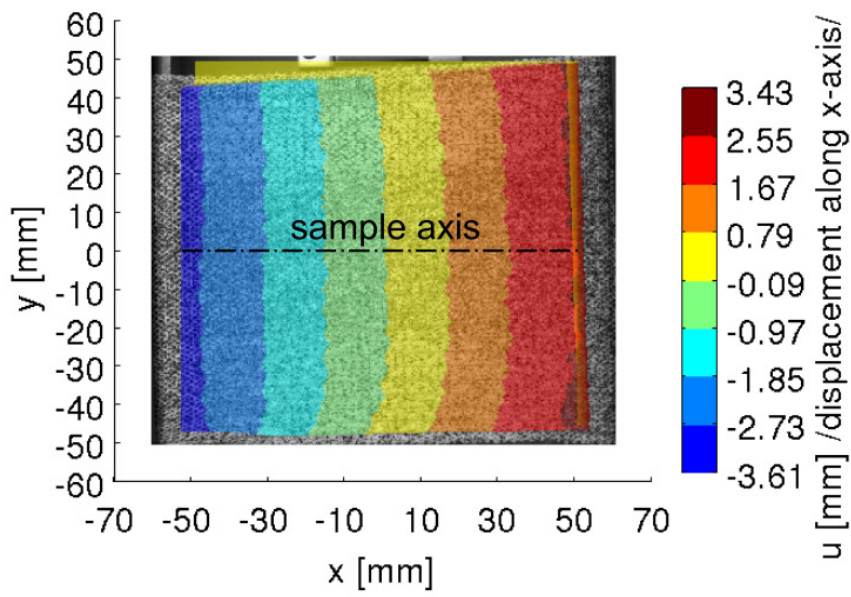

Figure 8. Field of displacements in the $x$ direction on the surface of the $\mathrm{NiTi}+\mathrm{PES}$ sample measured at loading instant 7 . The sample axis denotes the line along which the displacement $u$ is plotted in Figure 7 .

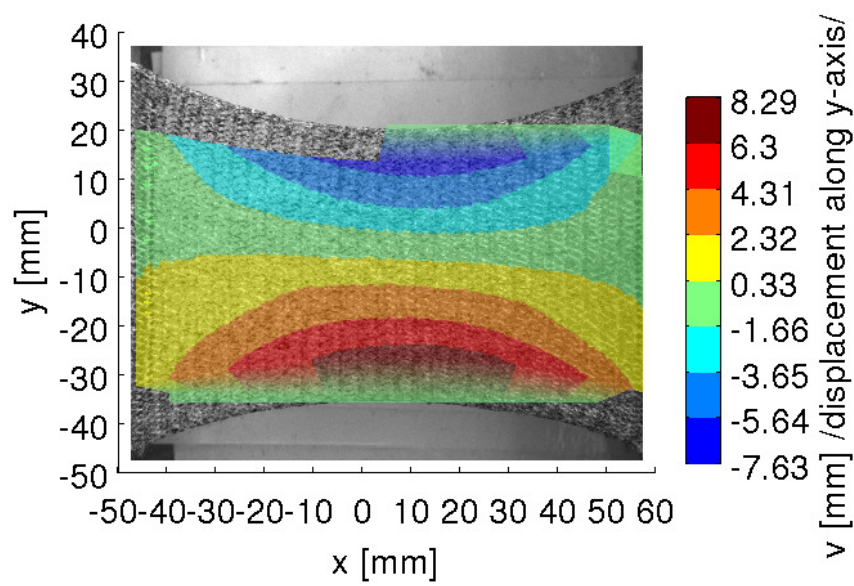

Figure 9. Field of displacements in the direction transverse to the loading axis measured on the surface of the PES sample and gathered at loading instant 2 (see Figure 6).

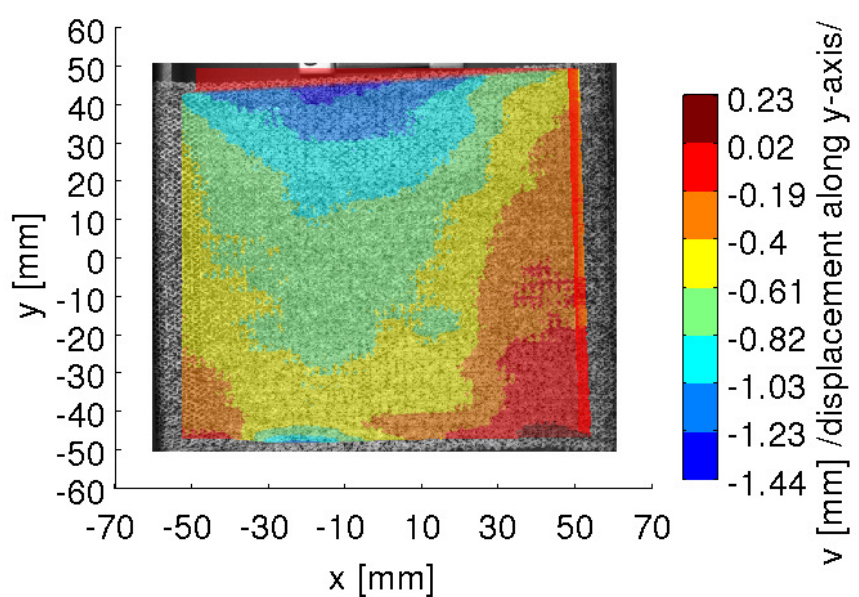

Figure 10. Field of displacements in the direction transverse to the loading axis and to the direction of the NiTi filaments placement measured on the surface of the $\mathrm{NiTi}+\mathrm{PES}$ sample and gathered at loading instant 7 (see Figure 6).

inhomogeneity was indeed measured as displayed in Fig. 7, showing displacement in the direction of the loading axis $x$ along the sample axis of the NiTi+PES sample (see Fig. 8) at several loading instants numbered according to Fig. 6.

Second, the tensile behaviour of the two samples differs considerably in terms of stretchability and stiffness. The
$\mathrm{NiTi}+\mathrm{PES}$ sample displays less stretchability, which is still greater than the reversible deformability of common metals. On the other hand, the NiTi+PES sample shows considerably greater stiffness. The tensile force needed to stretch the $\mathrm{NiTi}+\mathrm{PES}$ sample to $8 \%$ is more than 80 times greater than the PES sample. However, NiTi filaments affect the deformation properties not only in the direction of their placement but also in the transverse direction. By comparing displacements in the direction transverse to the loading axis measured on PES and $\mathrm{NiTi}+$ PES samples (see Fig. 9 and Fig. 10), one can conclude that the typical transverse shrinkage commonly seen on knitted textiles is considerably suppressed by NiTi filaments.

\section{Mechanical behaviour under bulging}

A reference PES sample free of NiTi filaments and two types of functional warp-knitted fabric samples were subjected to bulge tests. During these tests the samples were subjected to inner pressure loadings/unloadings in the regime of stroke of the pole-controlled ramps. The first tested functional warp-knitted fabric sample, denoted $\mathrm{NiTi}+\mathrm{PES} \# 1$, represents a single layer fabric with NiTi filaments inserted in the course direction as described above. There were 16 active NiTi filaments over the aperture of diameter of $100 \mathrm{~mm}$ of the bulge tester. The second tested functional warp-knitted fabric sample, denoted $\mathrm{NiTi}+\mathrm{PES} \# 2$, consists of two layers of $\mathrm{NiTi}+\mathrm{PES} \# 1$ mutually rotated by $90^{\circ}$ i.e. NiTi filaments were placed in two perpendicular directions.

As in the case of tensile testing, the pressure vs. the stroke of the pole characteristics of PES and NiTi+PES\#1,2 differ qualitatively as well as quantitatively (see Fig. 11, Fig. 12). $\mathrm{NiTi}+\mathrm{PES} \# 1,2$ characteristics inherit the typical non-linear hysteretic behaviour exhibited by NiTi filaments in tension. However, the plateau indicating the appearance of the transformation is not horizontal but inclined. This is apparently due to the fact that the macroscopic behaviour of the fabric is determined by a superposition of NiTi filament behaviour and that of the polyester weft-knitted structure. Moreover, it might be due to different active lengths of $\mathrm{NiTi}$ filaments depending on their placement over the aperture. Hence, individual NiTi filaments provide different transformation strains. Therefore, while some NiTi filaments are in the transformation stage, i.e., they project their plateaus on the overall mechanical behaviour of the fabric, other NiTi filaments are already in the elastic

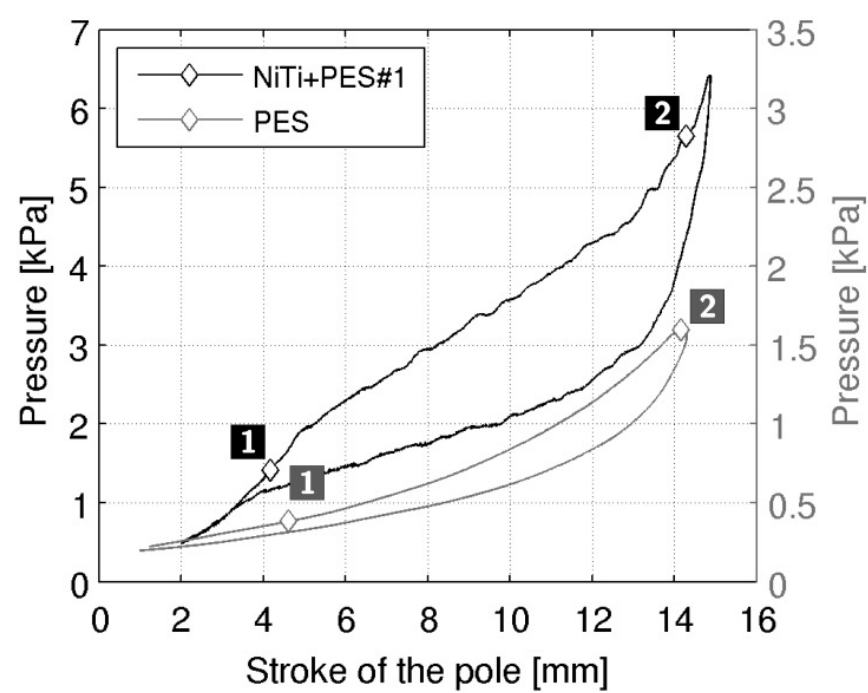

Figure 11. Pressure vs. stroke of the pole characteristics of the referential PES sample and one layer NiTi+PES\#1 sample both measured via the bulge tester. 


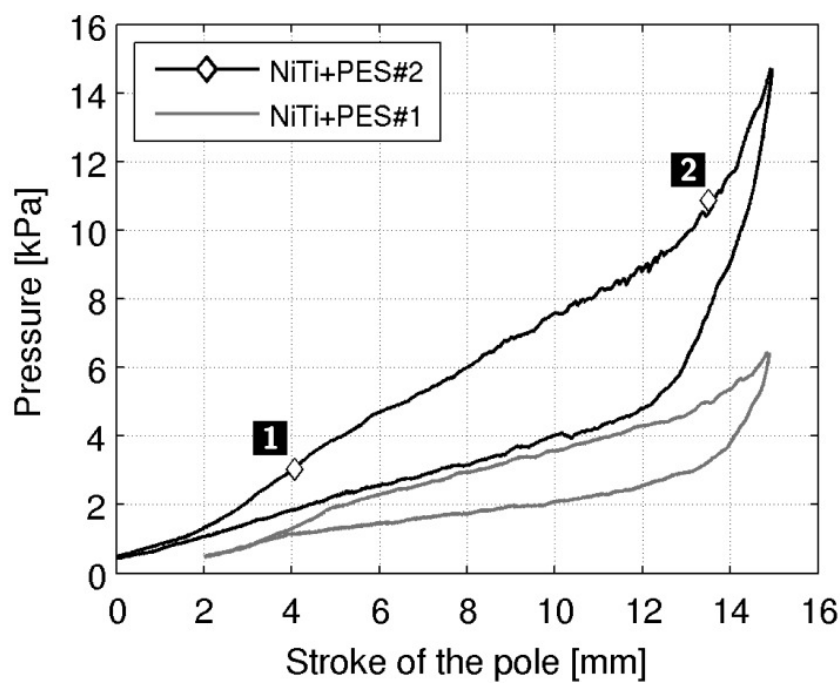

Figure 12. Pressure vs. stroke of the pole characteristics of the functional warp-knitted samples measured via the bulge tester.

regime beyond the plateau, i.e., they project linear elastic behaviour on the overall mechanical behaviour of the fabric.

Furthermore, $\mathrm{NiTi}+\mathrm{PES} \# 1,2$ show considerably greater stiffness while retaining the stretchability of PES. The pressure required to bulge the $\mathrm{NiTi}+\mathrm{PES} \# 1 / \mathrm{NiTi}+\mathrm{PES} \# 2$ sample to 15 $\mathrm{mm}$ of the stroke of the pole was nearly 6/12 times greater than for the PES sample. Note that the stroke of the pole of 15 $\mathrm{mm}$ was close to the limit of the reversible stretchability of all samples. As expected, the NiTi+PES\#2 sample containing two layers of the NiTi+PES\#1 sample exhibits double the stiffness of the NiTi+PES\#1 sample (see Fig. 12).

The responses of all the studied samples to bulging via inner pressure differ also in terms of the shape of the characteristic cap into which textiles are deformed during a bulge test. The $\mathrm{NiTi}+\mathrm{PES} \# 1$ sample exhibits a strongly inhomogeneous, nonaxisymmetric deformation field at low applied pressures, as illustrated in Fig. 13. The waviness of the deformation field is apparently driven by unidirectional distribution of NiTi filaments. On the other hand, the PES sample as well as the NiTi+PES\#2 sample show a homogeneous axisymmetric deformation field (see Fig. 14 and Fig. 15). Hence, it can be concluded that putting two differently oriented $\mathrm{NiTi}+\mathrm{PES}$ samples over each

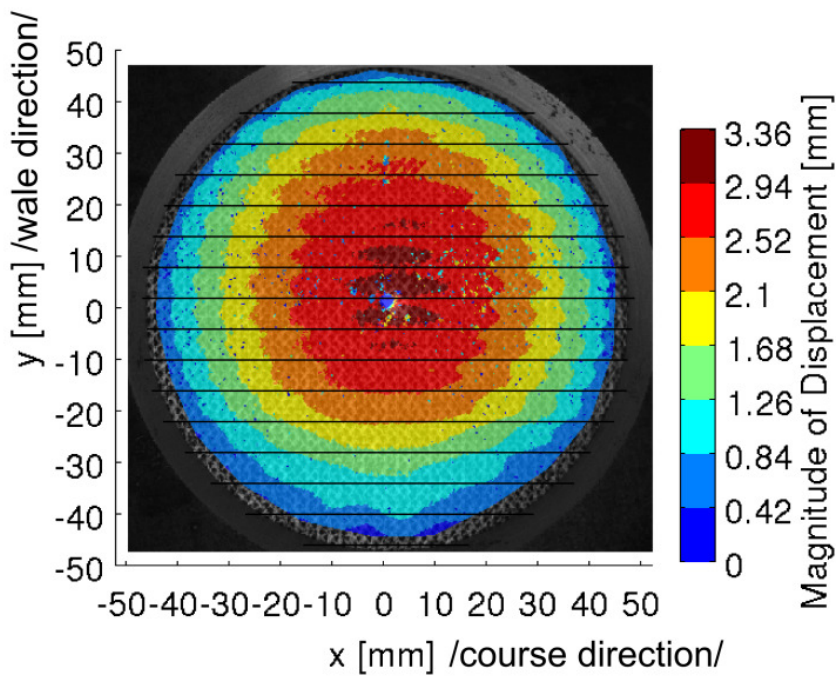

Figure 13. Magnitude of displacement of $\mathrm{NiTi+PES \# 1} \mathrm{sample} \mathrm{at} \mathrm{loading}$ instant 1 (see Figure 11) with indicated positions of NiTi filaments.

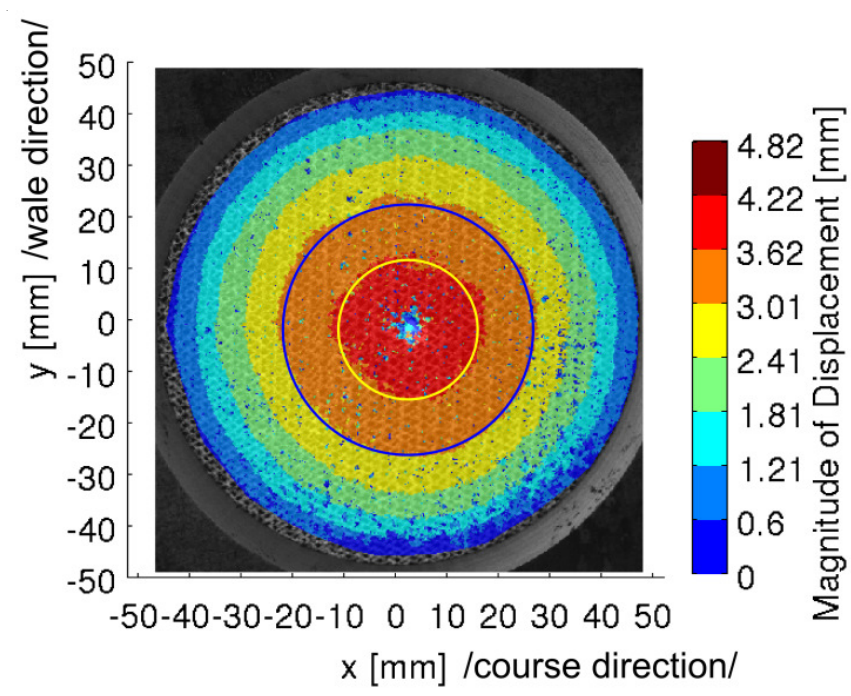

Figure 14. Magnitude of displacement of PES sample at loading instant 1 (see Figure 11) with indicated circular isolines.

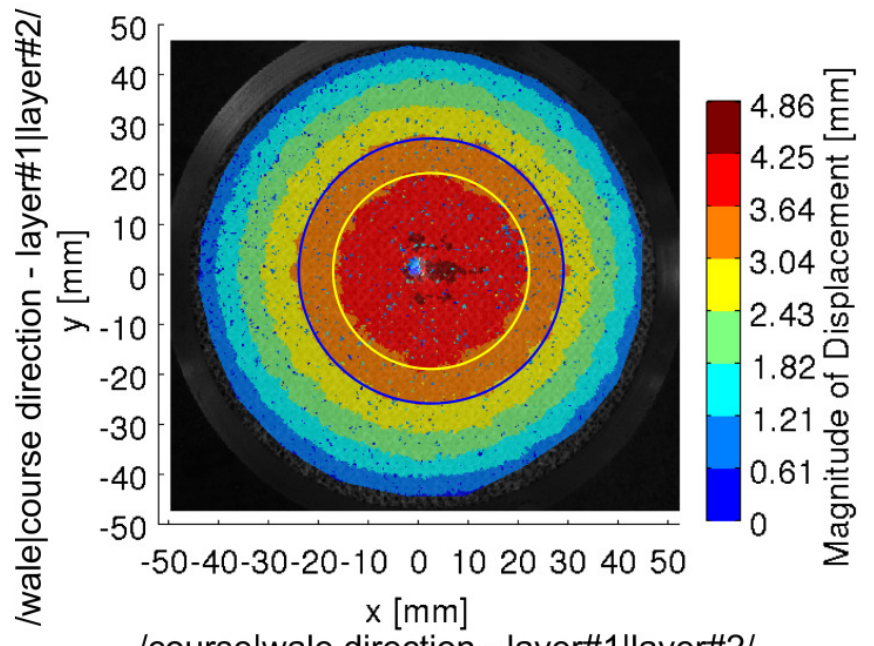

/course|wale direction - layer\#1|layer\#2/

Figure 15. Magnitude of displacement of $\mathrm{NiTi}+\mathrm{PES} \# 2$ sample at loading instant 1 (see Figure 12) with indicated circular isolines.

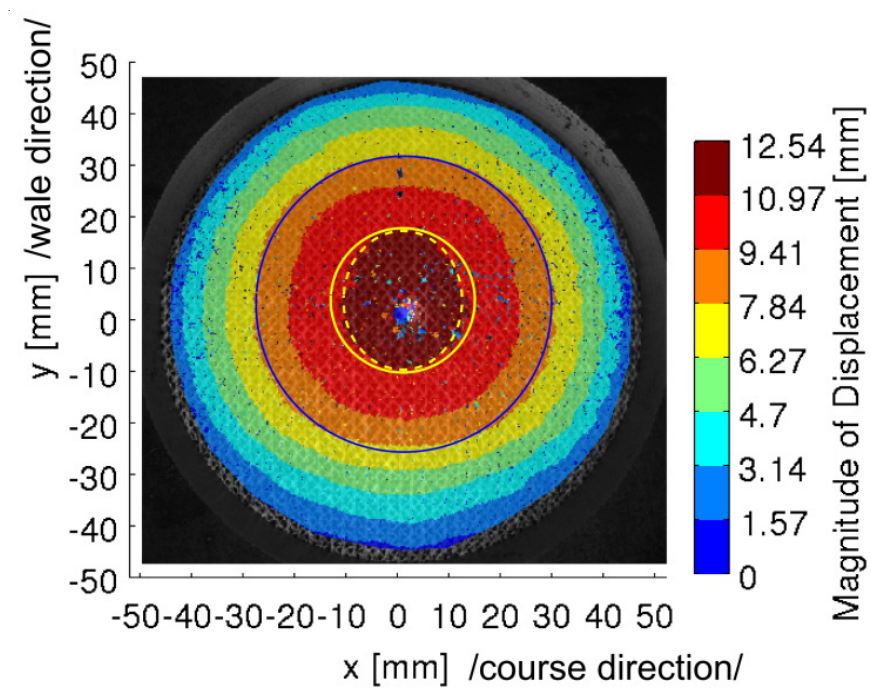

Figure 16. Magnitude of displacement of $\mathrm{NiTi}+\mathrm{PES} \# 1$ sample at loading instant 2 (see Figure 11) with indicated isolines and the asymmetry of the isoline at vicinity of the pole.

other is sufficient to homogenize the deformation induced by the applied inner pressure. 


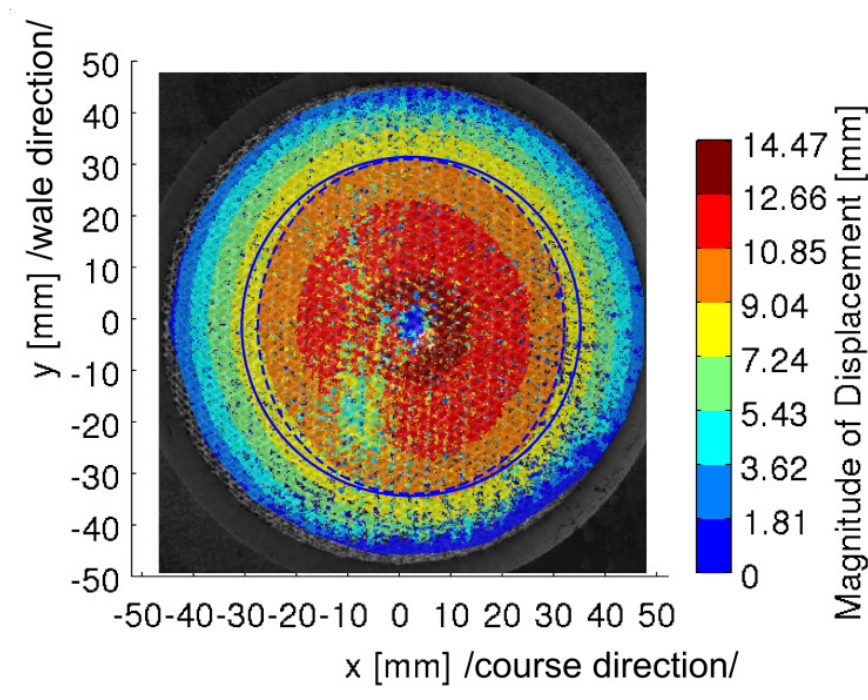

Figure 17. Magnitude of displacement of PES sample at loading instant 2 (see Figure 11) with indicated isolines and its asymmetry.

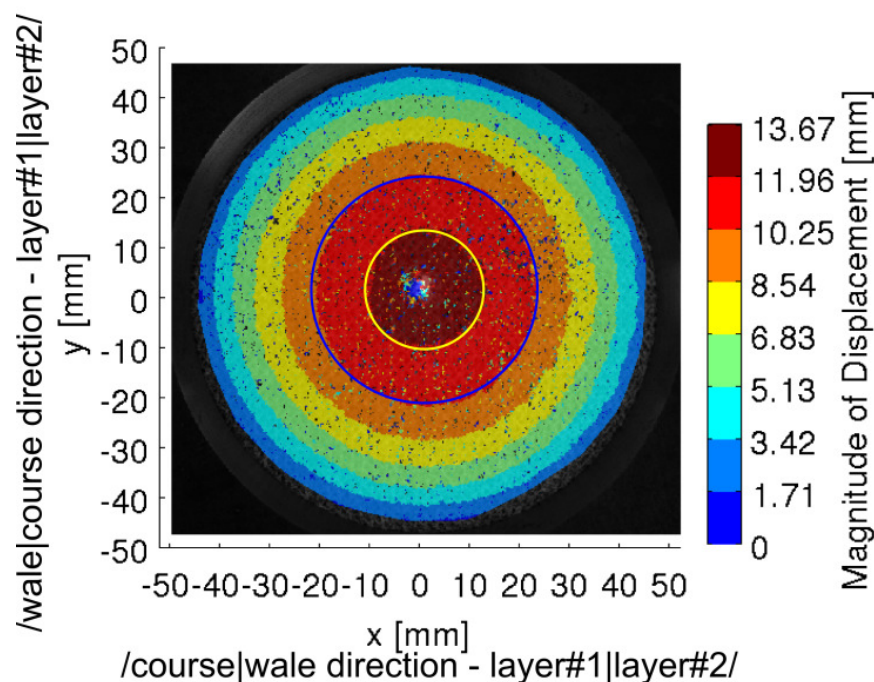

Figure 18. Magnitude of displacement of $\mathrm{NiTi}+\mathrm{PES \# 2}$ sample at loading instant 2 (see Figure 12) with indicated circular isolines.

With increasing applied pressure the deformation field of the $\mathrm{NiTi}+\mathrm{PES} \# 1$ sample tends to homogenize (Fig. 16). Moreover, higher applied pressures make the deformation of the $\mathrm{NiTi}+\mathrm{PES}$ 1sample and PES sample anisotropic (see elliptical isolines in Fig. 16 and Fig. 17). Apparently, this anisotropy is due to the structural deformation of the weft-knitted pattern as the isolines of both the samples exhibit the same elliptical shape and spatial orientation. On the other hand, the $\mathrm{NiTi}+$ PES\#2 sample exhibits a homogeneous isotropic deformation field at all applied pressures (see circular isolines in Fig. 15 and Fig. 18.) Therefore, we might conclude that an evenly oriented distribution of $\mathrm{NiTi}$ filaments makes the bulge deformation isotropic regardless of the structural anisotropy of the knitted fabric.

\section{Conclusions}

We designed and manufactured novel functional weft-knitted fabrics with integrated superelastic thin NiTi filaments. Their mechanical behaviour was analyzed using tensile and bulge tests. We analyzed the mechanical behaviour of reference polyester PES weft-knitted fabrics free of NiTi filaments, a single layer of the functional weft-knitted fabrics and two transversely oriented layers of the functional weft-knitted fabric. As an outcome of the experiments, we can summarize the mechanical properties of the functional weft-knitted fabric (FWKF) and the effect of NiTi filaments integration as follows:

- FWKF inherits the hysteretic mechanical behaviour from the behaviour of integrated NiTi filaments.

- FWKF sustains substantially larger tensile forces than the reference sample free of NiTi filaments.

- The tensile stretchability of FWKF is half that of the reference sample free of NiTi filaments.

- The deformation behaviour of FWKF under cyclic loadings is more stable and reversible than the reference sample free of $\mathrm{NiTi}$ filaments.

- FWKF shows considerably greater stiffness at bulge tests than the reference sample free of NiTi filaments, while retaining the stretchability of the reference sample.

- One can suppress the structural anisotropy of the weft-knitted pattern by combining several layers of FWKF with different $\mathrm{NiTi}$ filaments orientation.

\section{Acknowledgments}

We kindly acknowledge support from the Czech National Science Foundation provided through project no. P108/10/ 1296. This was published in the Ambience conference held in November 2011 at the Technical University of Boras, Sweden.

\section{References:}

1. Hu, J.; Shape memory polymers and textiles; CRC Press, Woodhead Publishing Limited: Boca Raton FL, Boston, MA, New York, and Washington, DC, 2007.

2. Budillon, F., Gries, T.; Shape memory textiles; Proceedings of SMST, October 3-7, 2004, Kurhaus Baden-Baden, Germany.

3. Chan Vili, Yvonne Y. F.; Textile Research Journal (May 2007) Vol. 77 No. 5 290-300.

4. Heller, L. et al.; E-MRS2007, European Physical Journal: Special Topics 158 (1).

5. Van Humbeeck, J.; Mat. Sci. Eng. A273-275 (1999) 134148.

6. Duerig, T., Pelton, D.; Mat. Sci. Eng. A273-275 (1999) 149160.

7. www.fwmetals.com 